\title{
Detection of amyloid in Alzheimer's disease with positron emission tomography using $\left[{ }^{11}\right.$ C]AZD2184
}

\author{
Svante Nyberg • Maria Eriksdotter Jönhagen • Zsolt Cselényi • Christer Halldin • \\ Per Julin • Hans Olsson • Yvonne Freund-Levi • Jan Andersson • Katarina Varnäs • \\ Samuel Svensson • Lars Farde
}

Received: 12 February 2009 / Accepted: 14 May 2009 / Published online: 5 June 2009

(C) The Author(s) 2009. This article is published with open access at Springerlink.com

\begin{abstract}
Purpose Current positron emission tomography (PET) radioligands for detection of $\mathrm{A} \beta$ amyloid in Alzheimer's disease (AD) are not ideal for quantification. To improve the signal to noise ratio we have developed the radioligand $\left[{ }^{11} \mathrm{C}\right] \mathrm{AZD} 2184$ and report here the first clinical evaluation. Methods Eight AD patients and four younger control subjects underwent 93-min PET measurements with $\left[{ }^{11} \mathrm{C}\right] \mathrm{AZD} 2184$. A ratio approach using the cerebellum as reference region was applied to determine binding parameters.

Results Brain uptake of $\left[{ }^{11} \mathrm{C}\right] \mathrm{AZD} 2184$ peaked within $1 \mathrm{~min}$ at $3-4 \%$ of injected radioactivity. AD patients had high radioactivity in cortical regions while controls had uniformly low radioactivity uptake. Specific binding peaked within $30 \mathrm{~min}$ at which time standardized uptake value ratios (SUVR) ranged between 1.19 and 2.57.

Conclusion $\left[{ }^{11} \mathrm{C}\right] \mathrm{AZD} 2184$ is a promising radioligand for detailed mapping of $A \beta$ amyloid depositions in Alzheimer's disease, due to low non-specific binding, high signal to
\end{abstract}

\footnotetext{
S. Nyberg $(\bowtie) \cdot$ Z. Cselényi $\cdot$ P. Julin $\cdot H$. Olsson $\cdot$ S. Svensson

L. Farde

AstraZeneca R\&D, Neuroscience Therapy Area,

15185 Södertälje, Sweden

e-mail: svante.nyberg@astrazeneca.com

M. E. Jönhagen · Y. Freund-Levi

NVS Department, Karolinska Institute,

Karolinska University Hospital Huddinge,

14186 Huddinge, Sweden

C. Halldin $\cdot$ J. Andersson $\cdot$ K. Varnäs $\cdot$ L. Farde

PET Centre, Department of Clinical Neuroscience,

Karolinska Institutet, Karolinska University Hospital,

Solna,

17176 Stockholm, Sweden
}

background ratio and reversible binding as evident from early peak equilibrium.

Keywords Alzheimer's disease - Imaging · Amyloid · Biomarker·PET · MRI

\section{Introduction}

Alzheimer's disease (AD) is a major cause of dementia estimated to affect more than 30 million people worldwide [1]. While clinical diagnosis is based on symptoms, definite $\mathrm{AD}$ diagnosis is based on post-mortem identification of extracellular $\beta$-amyloid $(A \beta)$ plaques and intraneuronal neurofibrillary tangles. Formation of neuritic plaques by $\mathrm{A} \beta$ deposits is thought to play a major role in the pathophysiology of $\mathrm{AD}$, and several therapeutic agents intended to remove or prevent the build-up of amyloid deposits are currently in clinical development. Recent development of positron emission tomography (PET) radioligands for detection of amyloid in patients has provided a new potential for diagnosis of $\mathrm{AD}$ during lifetime, ideally before the onset of clinical symptoms. It is also of interest to explore rate and distribution pattern of amyloid formation in relation to clinical progression. However, the first generation of radioligands for PET, including the Pittsburgh compound B ([ $\left.\left.{ }^{11} \mathrm{C}\right] \mathrm{PIB}\right)$ [2], are not ideal for quantification due to low signal to noise ratio, high non-specific binding or unfavourable kinetics [3].

We have recently developed the new putative radioligand, 5-(6-\{[tert-butyl(dimethyl)silyl $]$ oxy $\}-1,3$-benzothiazol-2-yl) pyridin-2-amine (AZD2184), which has high affinity in vitro for amyloid fibrils [dissociation constant (Kd) $8.4 \pm 1.0 \mathrm{nM}$ ] [4]. Autoradiography on cortical brain sections from APP/ PS1 mice and AD patients has shown that $\left[{ }^{3} \mathrm{H}\right] \mathrm{AZD} 2184$ has 
approximately 3 times higher signal to noise ratio than $\left[{ }^{3} \mathrm{H}\right]$ PIB, particularly because of lower non-specific binding [4]. Preclinical characterization thus suggests that ${ }^{11} \mathrm{C}$-labelled AZD2184 may provide higher sensitivity for $A \beta$ amyloid than the reference radioligand $\left[{ }^{11} \mathrm{C}\right] \mathrm{PIB}$. The aim of this first PET study with $\left[{ }^{11} \mathrm{C}\right] \mathrm{AZD} 2184$ in AD patients and control subjects was to evaluate the suitability of this radioligand for detection and quantification of brain $\mathrm{A} \beta$ amyloid. This report is a descriptive summary of the findings.

\section{Materials and methods}

The study was conducted at the PET Centre at Karolinska University Hospital Solna, with AD subjects recruited from the Karolinska University Hospital Memory Clinic at Huddinge, Sweden. The study was approved by the Regional Ethics Committee in Stockholm, Sweden and was performed in accordance with the Declaration of Helsinki and International Conference on Harmonization/Good Clinical Practice guidelines. Written informed consent was obtained from all subjects and caregivers before enrolment.

\section{Subjects}

Eight patients fulfilling DSM-IV AD criteria (four female, mean age: 62.6 years, range: $55-76$ ) were recruited. $\mathrm{AD}$ was characterized according to a routine clinical protocol including the Mini-Mental State Examination (MMSE), brain imaging (MRI or CT), neuropsychological assessments and CSF analyses. Four control subjects (male, mean age: 28.8 years, range: $23-36$ ) were recruited. To allow detailed examination of non-specific binding (i.e. the background), young control subjects were chosen to minimize the likelihood of amyloid pathology.

Properties and radiosynthesis of $\left[{ }^{11} \mathrm{C}\right] \mathrm{AZD} 2184$

$\left[{ }^{11} \mathrm{C}\right] \mathrm{AZD} 2184$ was produced according to Andersson et al. $[5] .\left[{ }^{11} \mathrm{C}\right]$ methane was produced in target via the ${ }^{14} \mathrm{~N}(\mathrm{p}, \alpha){ }^{11} \mathrm{C}$ reaction on nitrogen with $10 \%$ hydrogen, using $16.4 \mathrm{MeV}$ protons (GEMS PET trace cyclotron). $\left[{ }^{11} \mathrm{C}\right]$ methane was passed through a heated column containing $\mathrm{I}_{2}$ to produce $\left[{ }^{11} \mathrm{C}\right]$ methyl iodide via a radical reaction $[6] .\left[{ }^{11} \mathrm{C}\right]$ methyl iodide was trapped in a vessel containing $2.5 \mathrm{mg}$ of the precursor, 5-(6-(tert-butyldimethyl-silyloxy)benzo[d]thiazol2-yl)pyridin-2-amine), and potassium hydroxide $(10 \mathrm{mg})$ in dimethyl sulphoxide (DMSO) $(300 \mu 1)$. Heating at $125^{\circ} \mathrm{C}$ for 5 min was performed followed by deprotection with water $(200 \mu \mathrm{l})$ at room temperature for $2 \mathrm{~min}$ to yield $\left[{ }^{11} \mathrm{C}\right] 2184$. $\left[{ }^{11} \mathrm{C}\right] 2184$ was purified by reversed phase HPLC. The fraction that contained the product was evaporated to dryness and the residue re-dissolved into $6 \mathrm{ml}$ sterile physiological phosphate buffer $(\mathrm{pH}=7.4)$. After sterile filtration, the formulated product solution was sterile and free from pyrogens.

Imaging procedures and analyses

MRI was performed on a 1.5-T Siemens Avanto scanner. The PET system ECAT EXACT HR (resolution $3.8 \mathrm{~mm}$ full-width at half-maximum) was run in three-dimensional mode, using individually made plaster helmets for head fixation.

In each PET measurement radioactivity was recorded for 93 min following intravenous bolus injection of radioligand. Reconstructed, attenuation- and decay-corrected PET data were displayed as 47 transaxial slices in a $128 \times$ 128 matrix [7]. Arterial blood samples were drawn for determination of radioactivity in whole blood and plasma as well as for analysis of unchanged radioligand in plasma [7].

The first four AD subjects were examined once, while the other subjects were examined twice during the same day in a test-retest approach with at least $2.5 \mathrm{~h}$ between measurements. The injected radioactivity and specific radioactivity were

Table 1 Regional standardized uptake value ratios

\begin{tabular}{lll}
\hline Region & AD & CS \\
\hline ACG & $2.28(15.2)$ & $1.06(1.5)$ \\
CAU & $1.95(22.0)$ & $0.88(3.3)$ \\
CER & - & - \\
SWM & $1.50(17.6)$ & $1.33(7.2)$ \\
DLPC & $2.27(16.4)$ & $1.05(2.2)$ \\
DMPC & $2.37(16.9)$ & $1.02(3.1)$ \\
HIP & $1.31(17.3)$ & $1.05(11.5)$ \\
LTC & $2.23(15.3)$ & $1.06(1.6)$ \\
MTC & $1.54(15.1)$ & $1.07(4.1)$ \\
OCC & $1.74(18.8)$ & $1.15(2.4)$ \\
OFC & $2.42(14.3)$ & $1.05(1.1)$ \\
PAR & $2.12(15.6)$ & $0.99(4.4)$ \\
PONS & $1.19(16.3)$ & $1.12(5.9)$ \\
PCG & $2.57(16.5)$ & $1.07(9.2)$ \\
SMC & $1.59(17.0)$ & $0.97(9.0)$ \\
PUT & $1.88(19.3)$ & $1.07(5.1)$ \\
WB & $1.73(13.1)$ & $1.09(4.8)$ \\
\hline
\end{tabular}

Values shown are mean and coefficient of variation (\%), $n=8$ for AD, $n=4$ for $\mathrm{CS}$

$A C G$ anterior cingulate gyrus, $C A U$ caudate nucleus, $C E R$ cerebellum, $S W M$ subcortical white matter, $D L P C$ dorsolateral prefrontal cortex, $D M P C$ dorsomedial prefrontal cortex, HIP hippocampus, LTC lateral temporal cortex, MTC medial temporal cortex, $O C C$ occipital cortex, OFC orbitofrontal cortex, $P A R$ parietal cortex, $P O N S$ pons, $P C G$ posterior cingulate gyrus, $S M C$ sensorimotor cortex (primary), $P U T$ putamen, $W B$ whole brain 
Fig. 1 Summation PET images showing uptake between 7 and $90 \mathrm{~min}$ in SUV units

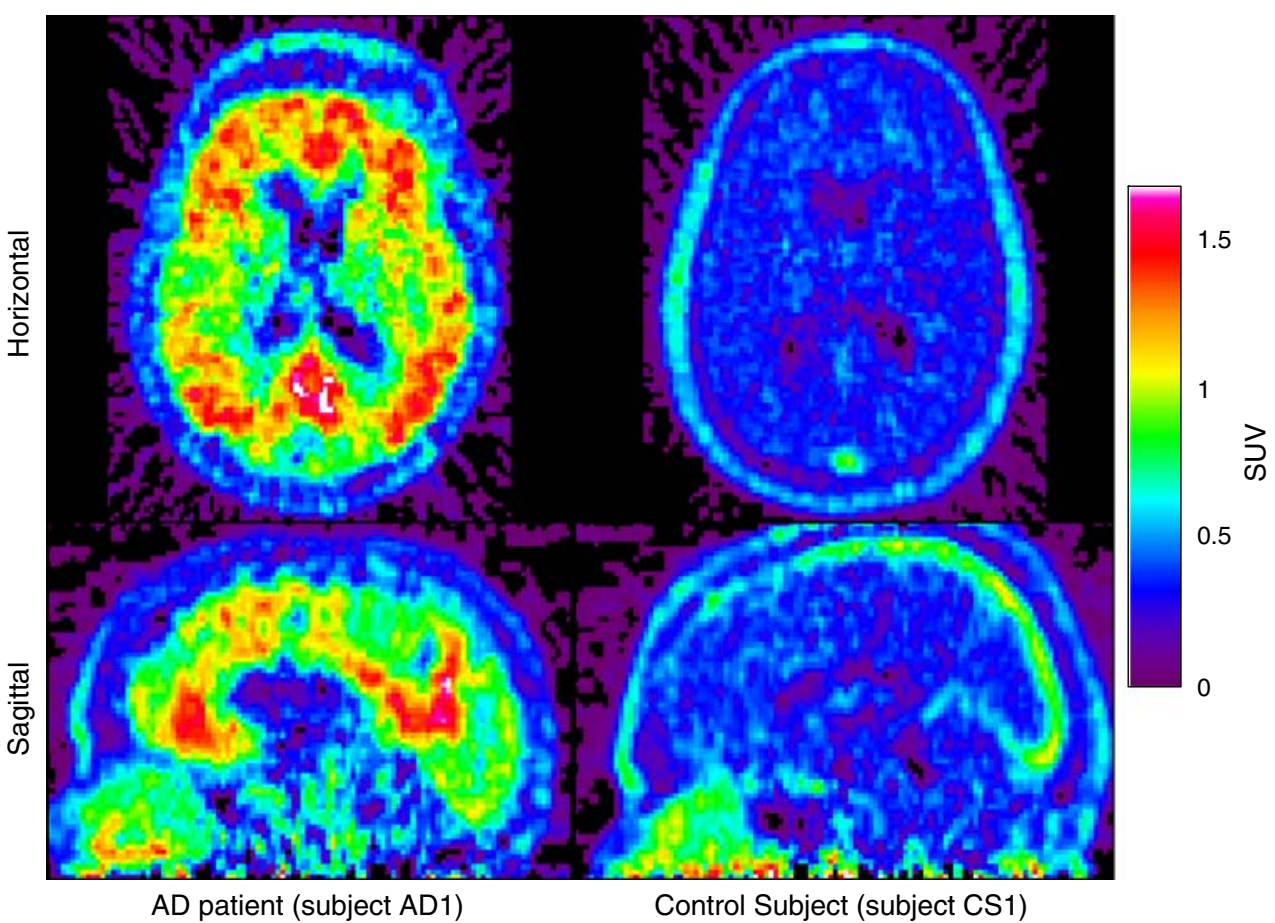

similar between $\mathrm{AD}$ patients $(323 \pm 10 \mathrm{MBq}$ and $14,866 \pm$

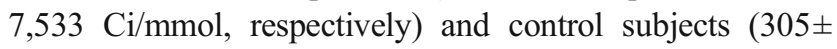
$46 \mathrm{MBq}$ and 29,083 $\pm 13,896 \mathrm{Ci} / \mathrm{mmol}$, respectively). Injected radioligand mass ranged from 0.04 to $0.48 \mu \mathrm{g}$. The MR images were reoriented in a standardized manner and used to segment the brain into grey and white matter. Regions of interest (ROIs) were drawn manually on the individual MR images for 17 cortical and subcortical structures (Table 1). The cortical ROIs were intersected with the grey matter segment image to only keep voxels within the grey matter. The PET images were coregistered to the reoriented T1-weighted MR images and time-activity curves (TACs) were extracted for the defined ROIs. No partial volume effect correction was applied, since such correction might overestimate uptake curves and specific binding in AD patients with atrophy.

The cerebellum was used as a reference region since this region is devoid of amyloid deposits [2, 8]. Specific binding was defined as the difference of radioactivity between other ROIs and the cerebellum. The standardized uptake value ratio (SUVR) has been proposed as an index for $A \beta$ amyloid binding [8]. The SUVR was calculated for the approximate time of peak equilibrium (24-30 $\mathrm{min}$ ).

\section{Results}

All subjects completed the study according to the protocol. The patients suffered from $\mathrm{AD}$ at a mild to moderate stage (median MMSE: 20, range: 16-26); seven were treated with choline esterase inhibitors. In all $\mathrm{AD}$ patients the summation PET images showed a high uptake of $\left[{ }^{11} \mathrm{C}\right]$ AZD2184 in regions known to contain $A \beta$ depositions, whereas uptake was low in the cerebellum (Fig. 1). In control subjects radioligand uptake was low and homogeneous. As evaluated from visual inspection, there was no difference in the normalized whole blood and the metabolite-corrected input function between $\mathrm{AD}$ patients and healthy controls (Fig. 2). Radioligand metabolism was fast with less than $20 \%$ of $\left[{ }^{11} \mathrm{C}\right] \mathrm{AZD} 2184$ remaining unchanged

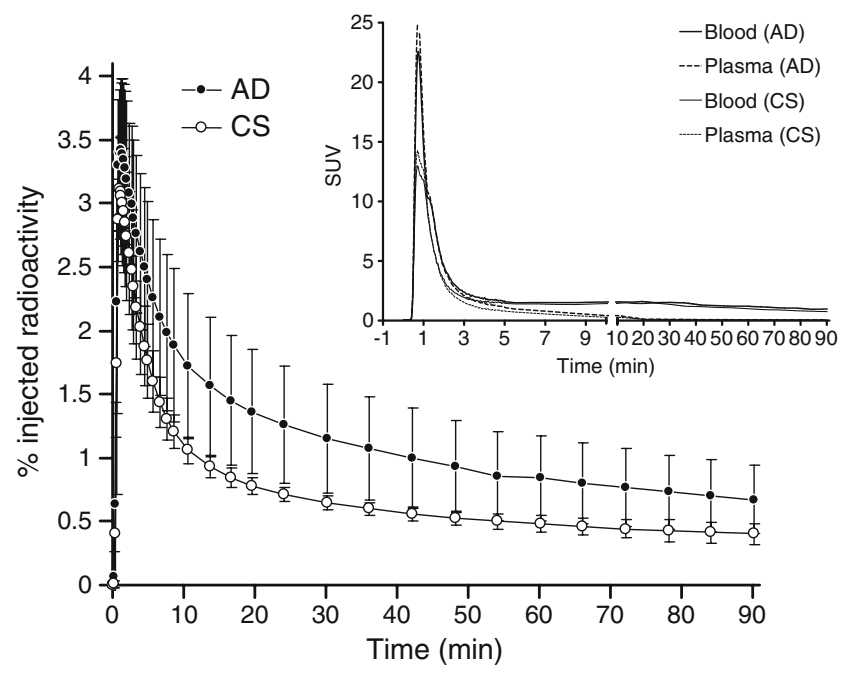

Fig. 2 Whole-brain relative uptake over time as measured by percentage of injected radioactivity that enters the brain. Insert shows the radioactivity in whole blood and the metabolite-corrected input function in $\mathrm{AD}$ patients and control subjects (mean $\pm \mathrm{SD}, n=8$ for $\mathrm{AD}$, $n=4$ for $\mathrm{CS}$ ) 
Fig. 3 Standardized uptake value over time for a cerebellum and $\mathbf{b}$ posterior cinguli. c SUVR over time. For clarity, the ratio of one is shown depicting no specific binding. d Approximated specific binding (i.e. radioactivity in ROI minus cerebellum) over time in posterior cingulate. Note the early peak of specific binding around $20 \mathrm{~min}(\mathrm{mean} \pm \mathrm{SD}$, $n=8$ for $\mathrm{AD}, n=4$ for $\mathrm{CS}$ )
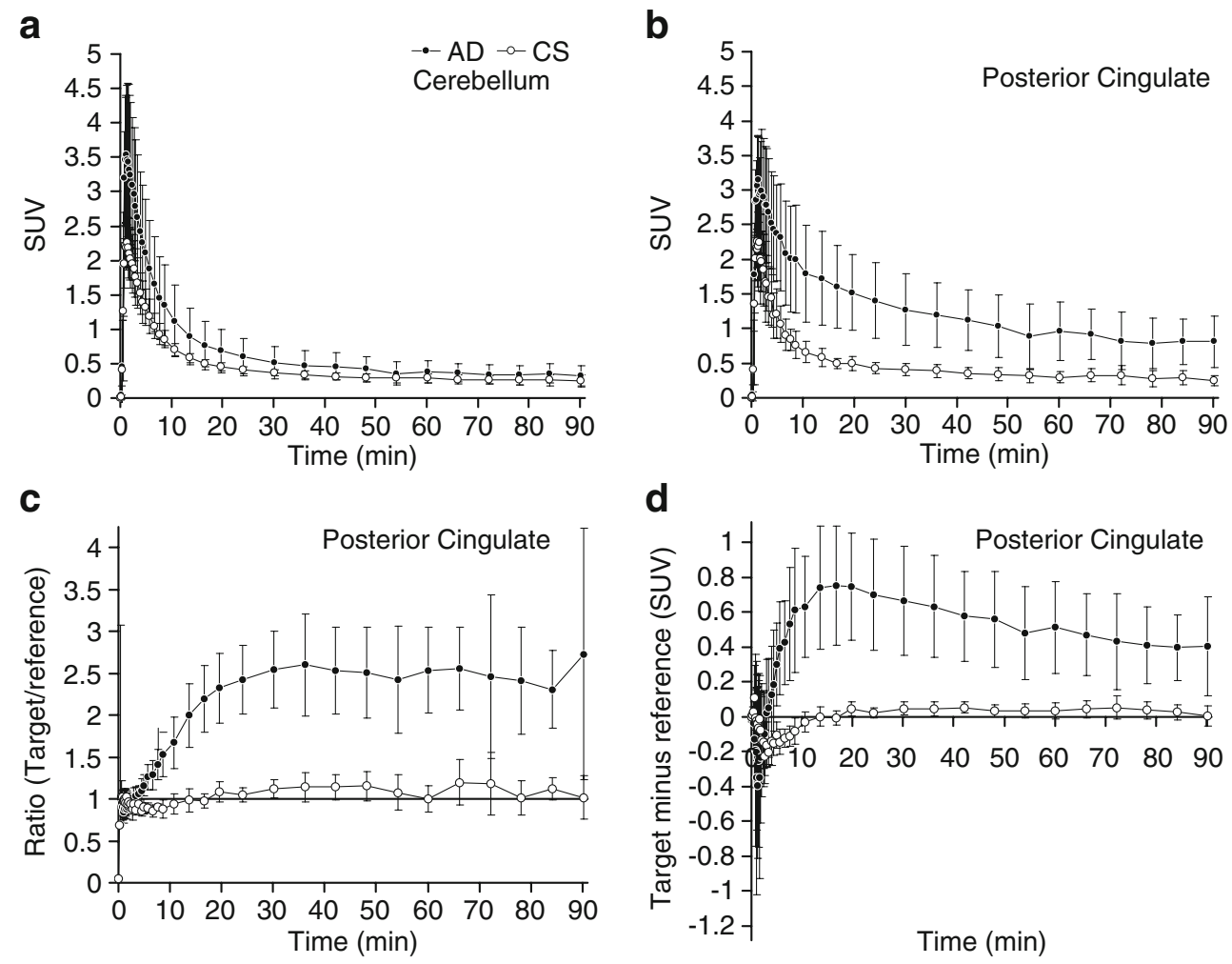

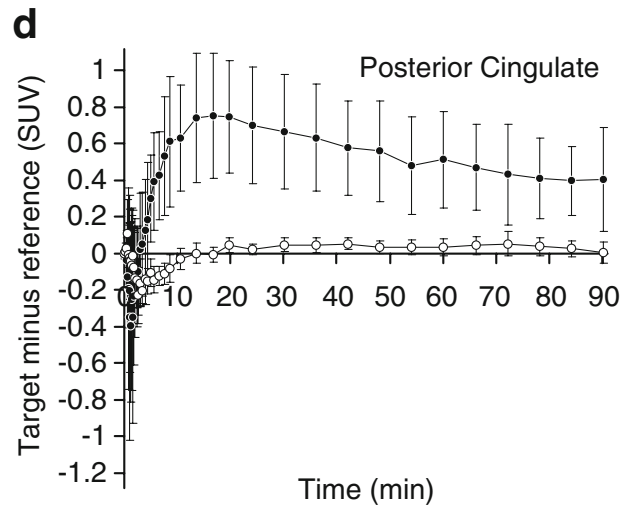

in plasma $20 \mathrm{~min}$ after administration. All metabolites were more hydrophilic than the mother compound.

The peak of whole-brain uptake of $\left[{ }^{11} \mathrm{C}\right] \mathrm{AZD} 2184$ appeared within 1 min and was between 3 and $4 \%$ of the injected radioactivity (Fig. 2). After the initial peak, there was a rapid washout of radioactivity in all subjects. TACs from $\mathrm{AD}$ patients showed markedly higher radioactivity in the target regions than in the cerebellum, whereas the TACs from control subjects were all on the same level. The cerebellar TACs were higher for AD patients than for the CS group in the early times of the experiment. However, from $40 \mathrm{~min}$ after injection the two curves were at a similar level (Fig. 3a).

The ratio of regional radioactivity to that of cerebellum was stable from $30 \mathrm{~min}$ after injection to the end of data acquisition (Fig. 3c). The difference between the TACs for the amyloidrich ROIs and that of the cerebellum gives an approximation of specific binding over time (Fig. 3d). All such curves from the $\mathrm{AD}$ patients peak at $20-30 \mathrm{~min}$ after injection and decline in the rest of the experiment. The curves from healthy volunteers were essentially flat as expected from the inspection of the raw regional TACs (Fig. 3d).

The SUVR were substantially higher for $\mathrm{AD}$ patients than for control subjects in regions expected to contain $A \beta$ depositions (Fig. 3c, Fig. 4). The control group had SUVR close to one in all regions and subjects (Fig. 4, Table 1). In a preliminary analysis (data not shown) the test-retest variability was on average $4 \%$ across cortical regions in all subjects.

\section{Discussion}

The aim of this first clinical study was to evaluate the suitability of $\left[{ }^{11} \mathrm{C}\right] \mathrm{AZD} 2184$ for detection of $\mathrm{A} \beta$ amyloid depositions in AD patients. Radioactivity was high in regions expected to contain $A \beta$ amyloid and low in the cerebellum, a reference region presumably devoid of amyloid deposits. The obvious inter-subject variability is consistent with expected differences in

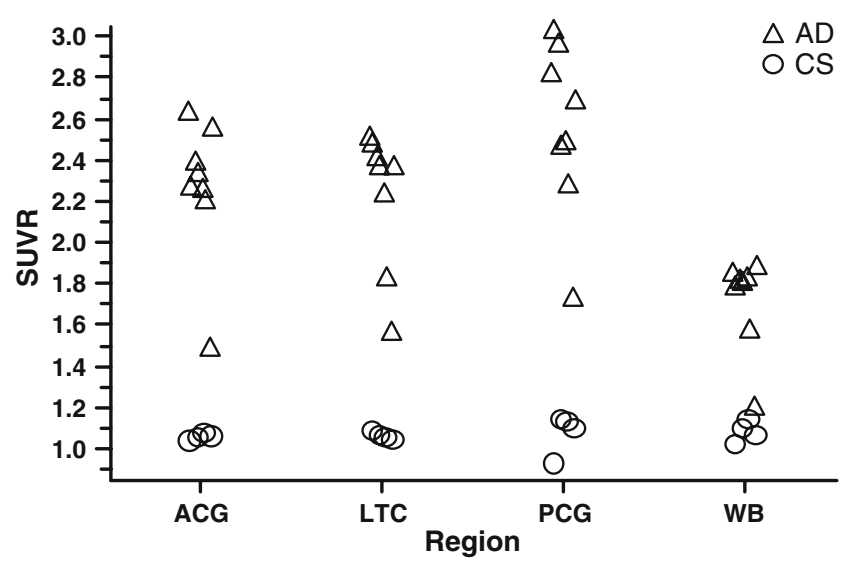

Fig. 4 Standardized uptake ratio values $(S U V R)$ for four different regions calculated using peak SUVR. $A C G$ anterior cingulate gyrus, $L T C$ lateral temporal cortex, $P C G$ posterior cingulate gyrus, $W B$ white matter 
plaque load and studies with other PET radioligands for amyloid [2]. In contrast, there was homogeneously low radioactivity across all brain regions in young controls subjects, likely to be devoid of amyloid depositions in brain. The PET results are consistent with preclinical demonstration of low background binding of $\left[{ }^{3} \mathrm{H}\right]$ AZD2184 [4]. The low background radioactivity is advantageous for detection of low levels of amyloid depositions such as before clinical onset of AD.

Maximal contrast (signal to noise) appeared already after about $30 \mathrm{~min}$, and specific binding peaked at about $20 \mathrm{~min}$. This indicates that the kinetics of $\left[{ }^{11} \mathrm{C}\right]$ AZD2184 binding are fast and reversible and may allow adequate quantification within $30 \mathrm{~min}$ after injection. The rapid washout may partly be related to the rapid metabolism of [11C]2184 in plasma. Short acquisition time improves patient compliance and allows for multitracer PET measurements.

The early high brain uptake of radioactivity (Fig. 2) demonstrates that the radioligand readily passes the bloodbrain barrier. Preclinical comparison between $\left[{ }^{3} \mathrm{H}\right]$ AZD2184 and $\left[{ }^{3} \mathrm{H}\right] \mathrm{PIB}$ in rodents and post-mortem AD brains supports the view that AZD2184 has advantageous contrast due to lower non-specific background binding [4]. Moreover, PET studies on $\left[{ }^{11} \mathrm{C}\right] \mathrm{PIB}$ have demonstrated slow kinetics and the need for long acquisition times for reliable quantification [9].

The current assessment does not include full kinetic analysis, which will be the subject of a more detailed report. The observation of high contrast images with low non-specific binding and the early peak of specific binding is, however, sufficient to conclude that $\left[{ }^{11} \mathrm{C}\right]$ AZD2184 is a promising radioligand for early detection and quantification of $A \beta$ amyloid depositions in the living human brain using short scanning protocols.

Open Access This article is distributed under the terms of the Creative Commons Attribution Noncommercial License which per- mits any noncommercial use, distribution, and reproduction in any medium, provided the original author(s) and source are credited.

Acknowledgements The authors gratefully acknowledge the efforts and support by all members of the Karolinska PET group, and the Stockholm Brain Institute. The study was funded in full by AstraZeneca R\&D, Södertälje, Sweden. In memoriam, Allan Johnson.

\section{References}

1. Wimo A, Jonsson L, Winblad B. An estimate of the worldwide prevalence and direct costs of dementia in 2003. Dement Geriatr Cogn Disord. 2006;21:175-81. doi:10.1159/000090733.

2. Klunk W, Engler H, Nordberg A, Wang Y, Blomqvist G, Holt D, et al. Imaging brain amyloid in Alzheimer's disease with Pittsburgh Compound-B. Ann Neurol. 2004;55:306-19. doi:10.1002/ana.20009.

3. Cai L, Innis RB, Pike VW. Radioligand development for PET imaging of beta-amyloid (Abeta) - current status. Curr Med Chem. 2007;14:19-52. doi:10.2174/092986707779313471.

4. Johnson A, Jeppsson J, Sandell J, Wensbo D, Neelissen J, Juréus A, et al. AZD2184: a radioligand for sensitive detection of betaamyloid deposits. J Neurochem. 2009;108:1177-86. doi:10.1111/ j.1471-4159.2008.05861.x.

5. Andersson J, Varnäs K, Cselényi Z, Gulyás B, Finnema S, Swahn B-M, et al. Radiosynthesis of an improved amyloid probe, [11C]AZD2184: PET characterization in the cynomolgus monkey and human brain. J Label Compd Radiopharm. 2009; Suppl 52 (in press).

6. Larsen P, Ulin J, Dahlstrom K, Jensen M. Synthesis of [11C] iodomethane by iodination of [11C]methane. Appl Radiat Isot. 1997;48:153-7. doi:10.1016/S0969-8043(96)00177-7.

7. Odano I, Halldin C, Karlsson P, Varonne A, Airaksinen AJ, Krasikova RN, et al. [18F]flumazenil binding to central benzodiazepine receptor studies by PET-quantitative analysis and comparisons with [11C]flumazenil. Neuroimage 2009;45:891902. doi:10.1016/j.neuroimage.2008.12.005.

8. Lopresti B, Klunk W, Mathis C, Hoge J, Ziolko S, Lu X, et al. Simplified quantification of Pittsburgh Compound B amyloid imaging PET studies: a comparative analysis. J Nucl Med 2005;46:1959-72.

9. Price J, Klunk W, Lopresti B, Lu X, Hoge J, Ziolko S, et al. Kinetic modeling of amyloid binding in humans using PET imaging and Pittsburgh Compound-B. J Cereb Blood Flow Metab 2005;25:152847. doi:10.1038/sj.jcbfm. 9600146 . 